\title{
Special Section Guest Editorial: Solid-state Lighting and LED Optics
}

\author{
Ching-Cherng Sun ${ }^{\mathrm{a}, \mathrm{b}}$ and Virendra N. Mahajan ${ }^{\mathrm{c}}$ \\ ${ }^{a}$ National Central University, Department of Optics and Photonics, Chung-Li, Taiwan \\ ${ }^{b}$ National Yang Ming Chiao Tung University, Department of Electrophysics, Hsin-Chu, Taiwan \\ ${ }^{c}$ University of Arizona, James C. Wyant College of Optical Sciences, Tucson, Arizona,
} United States

Light emitting diodes (LEDs) have been studied extensively for solid-state lighting (SSL) because of their fast response, vivid color, compact size, energy savings, and design freedom. Since the invention of high-brightness white LED in 1993, novel optical designs have made a big impact on higher lighting efficiency, more uniform illumination, higher contrast for special lighting, higher efficiency in light projection, and additional functions for new applications.

The call for papers for this special section was issued in November 2019 with paper submissions due by 1 July 2020. However, because of the COVID-19 pandemic and the resulting slowdown in research activity, the due date for paper submission was extended to 1 April 2021.

The published special section consists of six papers that highlight the recent advances in solving optical problems related to applications based on LEDs. Sun et al. introduce the current developments in optical design for the vehicle headlamps based on solid-state lighting. Their review article starts with an introduction of various regulations and focuses on the high-contrast cutoff line in the vehicle's low beams. Three optical design approaches, including a projection lens incorporated with a baffle or beam shaper, multi-segment reflectors, and complex lenses have been discussed comprehensively. A new insight called light-field management in designing a multi-segment reflector is discussed. Moreover, potential manufacturing errors and robustness of different optical approaches are analyzed. Finally, three approaches to performing the adaptive forward lighting which can provide a vehicle driver brighter and clearer vision without inducing glare to people on the roadway have been introduced.

Huang et al. have proposed a programmable ultraviolet LED (UVLED) array with collimating optics as a transfer lens with a light-field adjustable source. Its design shows that not only a uniform illuminance is available, but a specific illuminance distribution adapted to the feature of the exposed target can be achieved in the microlithography process.

Ying et al. report a design of the vehicle fog lamp that provides illumination in poor visibility. For vehicle lighting, front fog lamps using LEDs must provide a bar-shaped beam of light with a sharp cutoff at the top to increase the illumination directed toward the ground at a sharp angle. The authors have proposed and proved a new lens design based on a freeform surface responding to the requirements of the vehicle lighting. The design of the two-axis asymmetric freeform lens for the LED front fog lamp can meet ECE R19 class F3 regulation without using any other lens or reflector.

Near-infrared absorption spectroscopy (NIRAS) has been used to sense the state of blood glucose concentration through the strong absorption signal of blood glucose. Ding et al. have used the Henyey-Greenstein scattering skin model in a simulation to determine the light scattering. Accordingly, a dual-elliptical mirrors NIRAS system has been proposed to focus the LED light into increased depths of the human skin and collect more backscattered light. Such a mirror system can enhance the light collection efficiency by 1.6 times that of the conventional NIRAS system and 66 times that of the dual-elliptical lens NIRAS system.

The light pattern shaping by using a freeform lens on the illumination target is an important issue in LED optics. Liao et al. have proposed and demonstrated an effective design scheme for a freeform lens that includes a two-step process to direct appropriate flux to the expected target. The first step of the design is based on a point source, and then the surface of the freeform lens is

(C) 2021 Society of Photo-Optical Instrumentation Engineers (SPIE) 
determined. The flux deviation between the design and the actual target illumination becomes the factor in the second step when the point source is replaced by a real LED, and the angular flux compensation is applied to adjust the light pattern. Finally, the experimental results show that a high similarity between the target illuminance and its measurement value is observed and the full width at half maximum angle error is within $3 \%$.

Lee et al. have proposed adding a dual-wavelength LED to a white LED for underwater illumination to provide spectrum compensation at various illumination ranges so that the correlated color temperature (CCT) and the color rendering index (CRI) can be maintained at a requested level. Based on Mie scattering and Monte Carlo ray-tracing methods, the spectral transmission behavior of visible light in the seawater is estimated. The proposed dynamic light source can successfully maintain the CCT at $6000 \mathrm{~K}$ and the CRI above 80 under different underwater illumination distances.

In summary, this special section consists of six papers on SSL optical technologies addressing the applications of high-efficiency vehicle lighting, adaptive UVLED lithography, high-efficiency near-infrared absorption spectroscopy, freeform optics, and high-quality underwater illumination. These papers show that the scientific efforts keep improving human life in spite of the extraordinary circumstances resulting from the COVID-19 pandemic.

Ching-Cherng Sun joined the faculty of National Central University (NCU) in 1996 and became a chair professor in 2014. He is a fellow of SPIE and OSA, and president of Taiwan Photonics Society, vice president of Taiwan Information Storage Industry Association, and a board member of Taiwan Illumination Society. He was one of the chief editors of the Journal of Solid-state Lighting (Springer, 2014-2016), and associate editor of OSA's Applied Optics (2011-2017). He is currently an associate editor of OSA Continuum and an editorial member of Scientific Reports, and Crystals. He is co-chair of the SPIE conference on Current Developments in Lens Design and Optical Engineering, where he also chairs a session on solid-state lighting. He has received many awards, among them the Industry Contribution Award for University by the Ministry of Economic Affairs in 2009, Outstanding Technology to Industry and Outstanding Research Awards by the National Science Council in 2009, Outstanding Research Award by Taiwan Information Storage Association in 2012, Invention Award for Photonics by Far Eastern Y. Z. Hsu Science and Technology Memorial Foundation in 2014, Outstanding Research Award by the Ministry of Science and Technology in 2015, and the Engineering Award of Taiwan Photonics Society (TPS) in 2018. He has authored and co-authored more than 160 refereed papers. His current major research includes holography, holographic storage, photorefractive devices, optical system design, LED solid-state lighting, LED packaging, optical design, 3D scanning and modeling, and mixed-reality near-eye technology.

Virendra N. Mahajan is a graduate of the Wyant College of Optical Sciences, University of Arizona, where he is an adjunct professor. He is also an adjunct professor at the College of Optics and Photonics, National Central University in Taiwan. He has 40 years of experience working on space-based surveillance systems, the last 31 with The Aerospace Corporation, where he retired as a distinguished scientist/engineer. He is a fellow of SPIE, OSA, and a distinguished fellow of the Optical Society of India. He is a recipient of SPIE's 2006 Conrady award and 2015 Educator award. He is the author of Aberration Theory Made Simple, 2nd edition (2011), editor of Selected Papers on Effects of Aberrations in Optical Imaging (1994), and author of Optical Imaging and Aberrations, Part I: Ray Geometrical Optics (1998), Part II: Wave Diffraction Optics 2nd edition (2011), Part III: Wavefront Analysis (2013), and Fundamentals of Geometrical Optics (2014), all published by the SPIE Press. His book Aberration Theory Made Simple has been translated into Spanish, Russian, and Chinese. He is a chair of the SPIE conference on Current Developments in Lens Design and Optical Engineering. He is also an associate editor of OSA's Handbook of Optics, 3rd edition. 